\title{
TIPOS DE LISTA Y PERFILES PARLAMENTARIOS EN ESPAÑA Y ALEMANIA
}

\author{
CARLES PAMIES \\ Investigador en Ciencia Política \\ Universidad Autónoma de Madrid
}

TRC, núm. 45, 2020, pp. 557-566

ISSN $1139-5583$

\section{SUMARIO}

I. Tipos de listas electorales. II. Tipos de lista en perspectiva comparada: España y Alemania. III. Perfiles parlamentarios en España y Alemania. IV. Reflexiones finales.

\section{TIPOS DE LISTAS ELECTORALES}

Los procesos electorales suelen ser analizados por la Ciencia Política desde el lado de la demanda, en particular desde el comportamiento electoral. Incluso en sistemas con listas cerradas y bloqueadas como el español, se tiende a infravalorar la importancia de los factores de oferta en el funcionamiento de la democracia. Tanto en España como en muchos países europeos, particularmente después de la crisis de representación, es habitual hacer referencia a la necesidad de implementar cambios en los tipos de listas para democratizar los sistemas políticos ${ }^{1}$. Este interés en modificar las reglas electorales está relacionado con la voluntad de mejorar la capacidad de agencia del votante respecto a unos partidos que, a juicio de estos proponentes, habrían acaparado un poder excesivo en el proceso electoral. Estas modificaciones, a su vez, tienen también implicaciones relevantes en la democracia. Y aunque a veces estos efectos no son tan sustanciales como los legisladores suelen pensar ${ }^{2}$, la literatura sí muestra un mayor

1 Castro, D.A. La apertura de las listas electorales ¿un primer paso hacia la superación de la crisis de representatividad en la democracia española? En: Revista Española de Derecho Constitucional, no 112, 2018, pp. 115-144.

2 Bowler, S. y Donovan, T. The limits of electoral reform. Oxford: Oxford University Press, 2013. 
impacto de estas reformas en quién es elegido ${ }^{3}$, lo cual abre interrogantes sobre los efectos en la representación descriptiva en los parlamentos.

Por esta relevancia es por la que llama la atención que estén prácticamente ausentes del debate investigaciones que indaguen sobre la relación entre el modo de selección de los candidatos y las diferencias entre los perfiles sociodemográficos de los parlamentarios. La evidencia empírica muestra, a través de estudios de caso como el sueco, que los tipos de listas personalizadas favorecen a determinados perfiles, como los de aquellos candidatos con mayor nivel adquisitivo ${ }^{4}$, pero que las listas cerradas podrían favorecer a las mujeres ${ }^{5}$. Sin embargo, poco se sabe sobre los efectos de los tipos de lista sobre algunas de las características demográficas básicas de la población, como la edad, el origen nacional o la educación.

\section{Elementos formales en los tipos de lista}

Cuando hablamos de tipos de lista nos referimos a aquellas características del sistema electoral que tienen que ver con los distintos modos que tienen los votantes de articular sus preferencias, generalmente entre candidatos y partidos. Naturalmente, existen muchos modos de clasificar las distintas dimensiones en los sistemas electorales para establecer esas preferencias. Una tipología adecuada para el análisis de la personalización de los sistemas electorales y sus procesos de cambio es la propuesta por Renwich y Pilet para el caso europeo ${ }^{6}$.

Los autores establecen, en primer lugar, la dimensión del número de preferencias que se pueden expresar, es decir, si podemos votar a un solo candidato - $\mathrm{O}$ a un partido en las listas cerradas y bloqueadas del Congreso español- o si podemos votar a varios, como sucede en el Senado ${ }^{7}$. En segundo lugar, se distingue según el nivel de sofisticación en el apoyo a los candidatos. Aquí podemos diferenciar dos grandes modelos: un modelo categórico en el cual se apoya o no a un candidato determinado (el modelo usado en el Bundestag alemán) y un modelo ordinal en el que se establece un orden numérico sobre la preferencia (como el instant-runoff voting australiano y canadiense). En tercer lugar, existen modelos en los cuales solo se pueden establecer preferencias intrapartidistas o interpartidistas. Por modelos de preferencia intrapartidistas nos referimos a que todos los votos tienen que ir a candidatos de un mismo partido, como en los sistemas mayoritarios, mientras que por modelos interpartidistas se entiende aquellos en

3 Renwick, A. y Pilet, J. B. Faces on the Ballot. The personalization of electoral systems in Europe. Oxford: Oxford University Press, 2016, p. 240.

4 Nielsen, P. Ett decennium med personval. Erfarenheter och utfall. Stockholm: Norstedts Juridik ab, 2007.

5 Norris, P. Electoral engineering: Voting rules and political behavior. Cambridge: Cambridge University Press, 2004.

6 Renwick, A. y Pilet, J.B., cit., p. 240.

7 Las excepciones son Ibiza-Formentera, Menorca, Fuerteventura, El Hierro, La Gomera y la isla de La Palma. 
los que se puede votar a candidatos de distintos partidos, como el panachage suizo. En cuarto lugar, podemos diferenciar entre sistemas en los cuales hay una elevada cantidad de candidatos sobre los que escoger en relación con los candidatos «en juego» y aquellos en los que se escoge a pocos candidatos sobre el total de los que serán elegidos. Los sistemas de escrutinio mayoritario uninominal - first-pastthe-post - se sitúan en este último extremo, mientras que en Luxemburgo los votantes pueden asignar tantas preferencias como escaños. En quinto y último lugar, está la cuestión de la distancia entre el votante y el candidato, medida habitualmente por la magnitud del distrito. En este caso, los sistemas mayoritarios uninominales representan el polo de mayor cercanía con el representado, siendo su opuesto los sistemas de circunscripción única, como los Países Bajos.

En resumen, clasificar los sistemas electorales de acuerdo con estos parámetros es una tarea complicada que, además, refleja la complejidad y riqueza de los sistemas electorales alrededor del mundo, diferencias que, resultan aún más complejas si atendemos a los niveles territoriales. A esto último, y al estudio de caso, dedicaremos el siguiente apartado.

\section{TIPOS DE LISTA EN PERSPECTIVA COMPARADA: ESPAÑA Y ALEMANIA}

El caso español y el alemán resultan interesantes en un estudio comparado debido a sus diferencias en las reglas formales e informales en las elecciones a la cámara baja. Respecto a las reglas formales, estos dos casos muestran claras diferencias respecto a los tipos de lista. Ambos tienen un sistema de representación proporcional ${ }^{8}$, y aunque sus tipos de listas no están teóricamente en los extremos opuestos, dado que el sistema español es de listas cerradas y el alemán de listas mixtas - en concreto, un sistema de representación proporcional personalizada ${ }^{9}$ - las diferencias entre ambos modelos son importantes tanto sobre el papel como en la práctica. El modelo español forma parte de un grupo de familias minoritario a nivel comparado, que solamente comparte con un reducido grupo de países que incluye Portugal, Serbia, Israel, Rumanía y Bulgaria $^{10}$.

8 Cuando decimos que el sistema es proporcional nos referimos a sus características (como por ejemplo el hecho de ser plurinominales), no necesariamente a sus resultados. El caso español ha sido definido en ocasiones como un sistema proporcional con sesgos mayoritarios debido al prorrateo y al tamaño de las circunscripciones, tal y como explican Penadés, A. y Santiuste Cué, S. La desigualdad en el sistema electoral español y el premio a la localización del voto. En: Revista Española de Ciencia Política, n 32, 2013, pp. 89-116.

9 Fernández-Esquer, C. Los sistemas electorales de los Länder alemanes y sus reformas: panorámica general y tendencias de reforma. En: Revista española de derecho constitucional, vol. 39, $\mathrm{n}^{\circ}$ 117, pp. 101-135.

10 De Marcos, M.G. Listas desbloqueadas en los sistemas electorales autonómicos: ¿es necesario, posible y probable? En: M. PÉrez-Moneo y J. Vintró I Castells (coords.), Participación política: deliberación y representación en las Comunidades Autónomas. Congreso de los Diputados, 2017, pp. 377-404. 
En lo formal, existen similitudes tales como el número de preferencias (una, aunque en Alemania se vota en el circuito proporcional a partidos, Zweitstimme, y en el mayoritario a candidatos, Ersttimme) y el nivel de sofisticación (en ambos casos categórico). Al mismo tiempo, existen notables diferencias en los modelos de preferencias partidistas (interpartidistas solamente en Alemania según el circuito) y la distancia entre el votante y el candidato (elevada en España y reducida en Alemania). Más complicado es distinguir entre la relación entre candidatos votados y candidatos en juego, ya que técnicamente no existe un voto personalizado a candidatos en España.

La complejidad aumenta si descendemos al nivel regional, en particular en el caso alemán. Los sistemas electorales en los länder tienden a la representación proporcional, pero las similitudes terminan ahí. En realidad, existen cuantiosas particularidades en las fórmulas electorales, las barreras o los tipos de lista, con procesos de contagio tanto del sistema federal al subnacional como en sentido contrario $^{11}$. En lo relativo a las listas electorales, más de dos tercios de los Länder alemanes usan listas cerradas y bloqueadas, pero el resto utiliza fórmulas que van desde las listas abiertas con voto acumulativo, pasando por las listas desbloqueadas en Baviera y el sistema de Baden-Württemberg sin listas de partido. En España, por su parte, los sistemas son más homogéneos, y los contagios han tendido a ser del nivel regional al nivel nacional, como ilustran los casos de las reformas en la Región de Murcia en 2015, de Canarias en 2018 y en Aragón en 2019, que seguirían la lógica del papel de las Comunidades Autónomas como banco de pruebas de los cambios institucionales ${ }^{12}$.

Estas diferencias son relevantes no solamente en la literatura sobre las reformas de los sistemas electorales, sino también por sus efectos en la calidad democrática, tal y como lo recoge la literatura. En particular, este efecto del voto preferencial es observable sobre el tipo de candidatos escogidos en países como Austria, Bélgica, los Países Bajos, Irlanda y Malta ${ }^{13}$. Sin embargo, el efecto de los tipos de listas y de la personalización de los sistemas electorales no parece evidente para otras variables como la participación, la satisfacción con la democracia, o sobre la representación de las mujeres. El corolario parece ser el de que los legisladores en muchos países han tratado de hacer cambios grandes pero los votantes no se han dado por aludidos ${ }^{14}$.

\section{PERFILES PARLAMENTARIOS EN ESPAÑA Y ALEMANIA}

La literatura comparada apunta, tal y como hemos señalado, a que los electores votan distinto según el tipo de lista a la que se enfrentan en las urnas. En

11 FÉrnandez-ESQUer, C., cit., p. 105.

12 Montero, J.R. y Riera, P. Informe sobre la reforma del sistema electoral. Comisión de Estudios del Consejo de Estado, 2008.

13 Renwick, A. y Pilet, J.B., cit., p. 234.

14 Renwick, A. y Pilet, J.B., cit., p. 248. 
particular, se señala a la personalización de los sistemas electorales como un factor que lleva a una expresión más sofisticada de preferencias. Esto, a su vez, redunda en la elección de distintos tipos de candidatos.

El motivo de la comparación del caso español con el alemán recae en la ya señalada existencia de diferencias importantes en el tipo de lista para la Cámara Baja entre España y Alemania. No obstante, conviene señalar ciertas precauciones y algunas matizaciones. Respecto a las precauciones, en primer lugar, los sistemas en Alemania y España tienen muchas diferencias que van más allá del tipo de lista y que pueden estar detrás de los diferentes perfiles parlamentarios. En segundo lugar, no tratamos de decir que estas diferencias sean necesariamente las más importantes de todas las que afectan a los perfiles de los parlamentarios. En realidad, hay muchos otros elementos que pueden estar influenciando las características sociodemográficas de los parlamentos español y alemán. En tercer y último lugar, tampoco estamos estableciendo una relación de causa y efecto entre las configuraciones institucionales y los perfiles de los parlamentarios, sino que solamente tratamos de ver exploratoriamente si este tipo de hipótesis puede tener cierto recorrido futuro en el estudio de la representación política descriptiva y en los perfiles de los parlamentarios a nivel comparado.

Respecto a las matizaciones, conviene explicar que la comparación es siempre entre las cámaras bajas ${ }^{15}$ y que, por lo general, se han usado datos de encuesta en lugar de los datos oficiales por dos motivos: primero, porque no conocemos la existencia de bases de datos que crucen todas las variables escogidas (edad ${ }^{16}$, género, origen nacional y educación) y segundo, porque para poder argumentar que existen mecanismos informales distintos en ambos países hay que preguntar a los parlamentarios sobre cómo fueron elegidos, y eso solamente es posible mediante una encuesta.

Las bases de datos utilizadas son la base del Comparative Candidates Survey y la base de datos del proyecto CIUPARCRI ${ }^{17}$ «Ciudadanía y parlamentarios en tiempos de crisis y renovación: el caso comparado de España en el contexto del sur de Europa». Ambas bases utilizan un cuestionario básico conjunto básico, y sus encuestas tuvieron lugar en 2013 para Alemania y 2016 para España.

15 Cuando hagamos referencia a parlamentarios, nos referiremos siempre a los diputados de las cámaras bajas de ambos países, y nunca al Senado español ni al Bundesrat alemán.

16 Por la disponibilidad específica de datos de género, esta variable es la única para la cual se usarán los datos externos oficiales y de estudios.

17 Los resultados presentados en este artículo han sido obtenidos de las bases de datos del proyecto CIUPARCRI (Ciudadanía y parlamentarios en tiempos de crisis y renovación democrática. El caso comparado de España en el contexto del sur de Europa), financiado por el MINECO (Ministerio de Economía y Competitividad) con la clave CSO2016-78016-R. Las bases de datos del proyecto CIUPARCRI han sido construidas y actualizadas por los siguientes autores: Pablo Domínguez, Andrés Benítez, Manuel Portillo, Beatriz Carrasco, Elena González y Xavier Coller. El autor agradece a los propietarios de la base de datos por permitirle utilizarla en su investigación. 


\subsection{Elementos informales: inclusividad en la selección}

El primero de los supuestos que conviene comprobar es si, efectivamente, existen diferencias informales en el modo de selección de los candidatos en el Congreso español y el Bundestag alemán. Las diferencias informales las medimos por los niveles de inclusividad en la selección, una de las dimensiones básicas en la selección de candidatos ${ }^{18}$. Por inclusividad en la selección nos referimos al continuo de dos polos (inclusivo - exclusivo) según cuántas personas conforman el cuerpo que elige al candidato. Cuando los selectores conforman un grupo amplio hablamos de un proceso de selección inclusivo/incluyente, mientras que cuando hay uno o muy pocos selectores, hablamos de procesos exclusivos/excluyentes. Lo esperable es que las listas abiertas sean más inclusivas que las listas cerradas, debido a que en estas últimas el poder del partido es mayor y su influencia cabría esperar que se mostrara a la hora de escoger a los candidatos, reducto último del poder de los partidos ${ }^{19}$.

Los datos nos muestran que existen diferencias sustantivas entre el modo de selección en España y Alemania. Cuando son preguntados sobre qué fue más determinante a la hora de ser escogido en las listas, solo el 15\% de los parlamentarios españoles responde que fueron unas primarias (abiertas o cerradas para miembros), por casi un $80 \%$ en el caso de Alemania. En España, los candidatos son elegidos en su mayoría por los líderes de los partidos (un 58\%), mientras que en Alemania este modo de selección supone solo el 4,4\%.

Comprobada la existencia de diferencias en el plano informal, pasamos a ver los distintos perfiles en los parlamentarios españoles y alemanes. Las comparaciones de los distintos perfiles se articulan en cuatro grandes grupos: el género, la edad, el origen nacional y la educación. La elección de estos cuatro criterios se debe a que son variables de control habituales en la Ciencia Política en general, y también muy recurrentes en la literatura de representación política descriptiva en particular, especialmente los tres primeros. Para el género, se va a distinguir entre el género masculino y el femenino; para la edad, se utilizará cada año como valor discreto ${ }^{20}$; para origen nacional, se diferenciará entre aquellos que tenían la nacionalidad alemana o española al nacer y los que no, y respecto a la educación se distinguirá entre el nivel educativo (nivel máximo de estudios terminados) y los perfiles (qué disciplinas concretas). En todos los casos los valores perdidos ${ }^{21}$ han sido eliminados.

18 Rahat, G. y Hazan, R.Y. Candidate selection methods: An analytical framework. En: Party Politics, vol. 7, n' 3, 2001 pp. 297-322.

19 Gallagher, M. y Marsh, M. Candidate selection in comparative perspective: The secret garden of politics. Sage Publications, 1988.

20 El cuestionario no pregunta sobre la edad, sino sobre el año de nacimiento. Por este motivo, la variable ha sido recategorizada como la diferencia entre el año de la encuesta y el año de nacimiento.

21 Por valores perdidos hacemos referencia a las respuestas en blanco y a la categoría «no sabe». 
Antes de adentrarnos en los resultados, debemos partir de unas expectativas sobre la existencia de diferencias entre los dos países a nivel de sus perfiles.

Como hemos apuntado, en la literatura sobre las implicaciones en la representación política de los sistemas electorales y tipos de lista hay voces que defienden el efecto de las listas abiertas sobre la inclusión de mujeres ${ }^{22}$, pero el consenso está del lado de los beneficios de las listas cerradas para la representación de las mujeres ${ }^{23}$. Los argumentos que soportan estas afirmaciones son que, por un lado, las listas abiertas permitirían a los electores votar por mujeres, y que, por otro, las listas cerradas permiten a los partidos incluir cuotas en sus reglamentos para asegurar una composición equilibrada en género. La evidencia mayoritaria y más reciente estaría del lado de este último argumento, y el mecanismo sería que los parlamentarios que buscan la reelección (en inglés, los incumbents) suelen ser hombres $^{24}$, y que estos tienden a estar favorecidos por los votantes en sistemas con listas abiertas por una cuestión de familiariedad ${ }^{25}$. El argumento es reproducible al resto de perfiles. Por este motivo, en la medida en la que los parlamentarios suelen ser hombres, de mediana edad, de origen no extranjero y con niveles educativos altos, esperamos que estos perfiles aparezcan más en los sistemas con listas más abiertas ${ }^{26}$ : en nuestra comparación, el caso alemán.

\subsection{Perfiles parlamentarios: género, edad, origen nacional y educación}

La distribución de los parlamentarios en España y Alemania por género refleja de forma ajustada el efecto de las políticas de cuotas en la representación descriptiva. Las parlamentarias en España suponen un poco menos del mínimo legal de $40 \%$ que establece el artículo 44 bis de LOREG, si bien suponen entre el $51 \%$ y el $52 \%$ de la población ${ }^{27}$. En Alemania, en cambio, donde no existen

22 Taagepera, R. Beating the law of minority attrition. En: W. Rule y J.F. Zimmerman (eds.), Electoral Systems in Comparative Perspective: Their Impact on Women and Minorities. Westport, CT: Greenwood Press, 1994, pp. 235-245.

23 Ballington, J. Ten Years of Progress: Enhancing Women's Political Participation. En: Ten Years of Supporting Democracy Worldwide. Stockholm: International IDEA, 2005.

24 Gelman, A. y KING, G. Estimating incumbency advantage without bias. En: American Journal of Political Science, vol. 34, 1990, pp. 1142-1164.

25 MATLAND, R.E. Enhancing women's political participation: legislative recruitment and electoral systems. En: Ballington, J. y Karam, A. (Eds.), Women in parliament: Beyond numbers. Stockholm: International IDEA, 2005, pp. 93-111.

26 El objetivo aquí no es el de determinar que las listas abiertas en su conjunto tienden a resultar en perfiles más continuistas de candidatos, puesto que el desempeño de los sistemas cerrados depende de muchas variables, entre ellas la voluntad de los partidos. Solo tratamos de decir que, en nuestra comparación, el sistema alemán debería premiar más a los perfiles ya existentes.

27 Los datos comparativos para el género y las demás variables provienen de Eurostat. En su mayoría, del informe «People in the EU: who are we and how do we live?» de 2015, disponible en: https://ec.europa. eu/eurostat/documents/3217494/7089681/KS-04-15-567-EN-N.pdf 
tales cuotas de género, el porcentaje de mujeres se reduce a un $36,9 \%$ frente a un $63,1 \%$ de hombres ${ }^{28}$.

En lo que respecta a la edad, las diferencias se reducen un poco entre los dos países $^{29}$. En España, los parlamentarios son algo mayores que los alemanes, aunque la diferencia es solo de algo más de dos años. En España la media es de 48,38 años y en Alemania de 45,91. Sí vemos más diferencias en el modo en el que se distribuyen las edades. Los parlamentarios alemanes se reparten por edad de forma más homogénea que los españoles. Dicho de otro modo: hay más parlamentarios jóvenes y mayores en Alemania. En concreto, en España ningún parlamentario que haya respondido a la encuesta tiene menos de 30 años ni más de 67 , mientras que en Alemania han respondido parlamentarios de entre 18 y 79. Todo esto teniendo en cuenta que las pirámides de población alemana y española son bastante similares. Los parlamentarios en España se agrupan más en el centro (casi el 60\% tiene entre 40 y 55 años), mientras que el $22 \%$ de los parlamentarios alemanes tienen menos de 35 años, por solo un $9 \%$ de los españoles. Finalmente, los parlamentarios españoles con 60 años o más suponen solo un $5 \%$ de la muestra, por un $11 \%$ de los parlamentarios alemanes. Esto contrasta con el hecho de que la población de más de 65 años supone el 15\% de la población española en 2014 y el $18 \%$ en Alemania ${ }^{30}$.

En términos de origen nacional, los parlamentarios en España y Alemania son, en su práctica totalidad, españoles y alemanes de origen. Al ser preguntados, el cuestionario hace referencia al origen de dos modos. En primer término, se les pregunta si eran nacionales del país desde su nacimiento, siendo ese el caso del 98\% de los parlamentarios españoles y el 95\% de los alemanes. En la población, las personas de origen extranjero suponen alrededor del $10 \%$ en ambos países en 2016, usando datos de Eurostat ${ }^{31}$. Sin embargo, si desgranamos por el país de origen vemos que casi 20 parlamentarios alemanes eran nacionales de la República Democrática Alemana, por lo que las diferencias a nivel de origen quedan prácticamente igualadas.

Por último, a nivel de educación — medida como el nivel educativo máximo alcanzado- los parlamentarios españoles tienen niveles más altos que los parlamentarios alemanes. Esto se mantiene así incluso si tomamos como referencia las

28 En las últimas elecciones de 2017 la infrarepresentación de mujeres en el Bundestag alemán cayó hasta un $31 \%$.

29 De aquí en adelante los datos son de encuesta, por lo que podría haber pequeñas variaciones en los resultados y la representación real en los parlamentos debido al muestreo y a otras cuestiones de naturaleza estadística.

30 La comparación entre la población y los parlamentos no es directa en la medida en la que los menores de 18 años no pueden ser electos, ni tampoco es habitual retrasar la edad de jubilación por encima de los 70 años.

31 Por origen extranjero aquí se hace referencia a personas que nacieron en un país distinto al que se presentaron como candidatos, mientras que para la pregunta a los candidatos se pregunta sobre la nacionalidad. 
tres categorías de educación más elevadas, para dar cuenta del mayor reconocimiento social de la formación profesional en Alemania. La brecha fundamental en el nivel educativo de los parlamentarios españoles y alemanes se encuentra en las categorías de estudios secundarios y estudios universitarios finalizados. Sin embargo, entre España y Alemania existen diferencias en la población en el nivel educativo máximo alcanzado. En particular, el 24\% de los alemanes tiene estudios de ciclo superior (universitarios o no), frente a un 32\% en España, usando datos de 2015. Las diferencias entre la suma de las tres categorías superiores de cada país muestran una sobrerrepresentación real de 57 puntos en Alemania y de 64 en España.

Las diferencias entre los perfiles educativos de los parlamentarios españoles y alemanes existen también en las disciplinas. En España, el perfil mayoritario es el de las ciencias jurídicas (licenciados y graduados en Derecho), mientras que en Alemania el perfil mayoritario es el de las ciencias naturales (como las Ciencias de la Salud, Física o Biología) y formales (como Ingenierías, Matemáticas o Arquitectura). Por su parte, las Humanidades son un perfil más común en Alemania, destacando la Educación y la Historia. Finalmente, los perfiles de Ciencias Sociales son los segundos más comunes en cada uno de los países, con alrededor del $30 \%$ de los candidatos con estudios superior en esa especialidad.

\section{REFLEXIONES FINALES}

A lo largo de estas páginas hemos desgranado los distintos tipos de listas electorales y hemos comparado el sistema de representación proporcional personalizada alemán con el sistema de listas cerradas y bloqueadas español. Los tipos de lista, tal y como hemos argumentado, son relevantes no solamente como una forma de analizar descriptivamente los sistemas electorales, sino por sus implicaciones y efectos sobre las democracias.

Los sistemas de listas español y alemán presentan diferencias tanto a nivel formal como informal. Las diferencias formales incluyen los modelos de preferencia y la distancia entre el votante y el candidato, mientras que en las informales vemos mayores niveles de inclusividad en la selección en los candidatos alemanes, que tienden a ser escogidos por procesos de primarias, confirmando nuestras previsiones de que las listas cerradas son más inclusivas en su selección de candidatos.

En cuanto a los efectos sobre la representación política, hemos analizado las diferencias en los perfiles de los parlamentarios. Aquí, los resultados son variados respecto a nuestras sospechas, ya que el sistema de listas cerradas español acerca sus resultados a los de la población general en el caso del género, pero no lo hace para la edad y el nivel educativo. En el caso del origen nacional, ambos sistemas son igual de poco representativos de la población de origen extranjero, en particular si incorporamos a los nacionales de la República Democrática Alemana.

En resumen, aunque no podemos establecer una relación causal, parece que los tipos de lista podrían tener efectos en qué tipo de candidatos son elegidos. Sin 
embargo, los perfiles también podrían estar determinados por reglas formales como las cuotas en el caso del género o las reglas informales, como algunas de las estrategias propias de los partidos a la hora de escoger sus candidatos.

Los estudios posteriores en esta línea deben tener en cuenta de forma comparada la relación entre los determinantes de los sistemas electorales y los perfiles demográficos de los parlamentarios, poniéndolos en relación con la población, a fin de dar cuenta de las dinámicas de representación política y su interacción entre las normas formales e informales en la selección de candidatos.

$$
* * *
$$

\section{TITLE: Electoral lists and parliamentary profiles in Spain and Germany}

ABSTRACT: Electoral lists are a key element in electoral systems, particularly because of their implications for the functioning of democracy. However, it is not usual to find studies on how these attributes may be related to the type of candidates included in the electoral lists. We start with an overview of the different types of lists and we portray the cases of Spain and Germany - two different systems in both their formal and informal rules. After that, we use the comparative data of a transnational European survey to see the differences in the profiles of the parliamentarians in the lower houses of both countries. We firstly expected that a more open list system - the German case - would be more inclusive in terms of selection, which was confirmed. We also expected our close-list system in Spain to be more inclusive in parliamentary profiles, and this was the case only for gender, while in terms of age and educational levels and profiles was not. These results allow new inquiries to further research on the relationship between formal and informal factors of candidate selection and demographic representation profiles.

Resumen: Los tipos de listas son un elemento central en los sistemas electorales, en particular por sus implicaciones en el funcionamiento de la democracia. Sin embargo, no es habitual encontrar estudios sobre cómo esos atributos pueden estar relacionados con el tipo de candidatos que se incluyen en esas listas. Tras una panorámica general de los distintos tipos de listas y la justificación de los casos de España y Alemania — dos sistemas distintos tanto en sus reglas formales como informales-. Tras esto, usamos los datos comparados de una encuesta trasnacional de ámbito europeo para ver las diferencias en los perfiles de los parlamentarios en las cámaras bajas de ambos países. Nuestra primera expectativa era encontrar mayor inclusividad en la selección en sistemas de listas más abiertas como el alemán, lo cual se confirma. Paralelamente, nuestra expectativa era que un sistema de listas cerradas como el español favorecería mayor inclusividad en los perfiles parlamentarios, pero esto es solo para el caso del género, mientras que ocurre lo contrario para edad y para el nivel y el perfil educativo. Estos resultados abren una vía de investigación sobre la relación entre los factores formales e informales de la selección de candidatos y los perfiles demográficos de representación

KEY WORDS: closed-lists, preferential voting, electoral system, representation, candidates

PalABRAS Clave: listas cerradas, voto preferencial, sistema electoral, representación, candidatos

FECHA DE RECEPCIÓN: 28.01.2020

FECHA DE ACEPTACIÓN: 19.02.2020 